\title{
Flow field analysis of multi-stage middle-open centrifugal pump inter-stage flow passage
}

\author{
Xiao Zhang ${ }^{1, *}$, He Huang ${ }^{2}$ \\ ${ }^{1}$ Zhenjiang Campus, Army Military Transportation University of PLA, Zhenjiang 212000, China \\ ${ }^{2}$ School of Traffic Engineering, Nanjing Institute of Industry Technology, Nanjing 210046, China
}

\begin{abstract}
In order to study the flow velocity, static pressure and turbulent kinetic energy distribution of the inter-stage flow passage, the numerical calculation of the inter-stage flow passage of the multistage split centrifugal pump was carried out under the design condition. The results show that the fluid flows along the inter-stage water flow channel, and backflow and vortices are generated at the guide vanes at the end of the bridge, which causes certain energy loss. In this paper, based on the original design, three different improvement schemes are proposed by changing the shape and position of the guide vane for the backflow and vortex generated near the guide vanes. The improved scheme is numerically simulated, and the energy loss values of the four different flow passages are calculated by integration. After comparison and analysis, the second scheme is determined as the best scheme, and the accuracy of simulation is verified by experiments..
\end{abstract}

With the rapid development of the national economy, the gradual deterioration of natural conditions and the shortage of water resources, the demand for high-efficiency and lowenergy multistage middle-open centrifugal Pumps is increasing year by year in various industries. Compared with the traditional multistage centrifugal pumps, it has a larger flow, higher head and power as well as the efficiency ${ }^{[1-3]}$, which meets the needs of China's economic development. At present, there is no mature theory for the design of multistage middle-open centrifugal pumps in China, and there are many problems in the domestic one, the efficiency is much lower than that of imported pumps ${ }^{[4-5]}$. So how to improve the efficiency is the main research direction of multistage middle-open centrifugal pump. Its internal structure is complicated, and the flow channel between the two stages is seriously distorted. Therefore, by studying the flow field of the multi-stage pump flow channel, analyzing the factors that affect the performance of the pump and improving the structure are important methods to improve the overall performance and efficiency of the pumps ${ }^{[6-8]}$.

In this paper, the flow field of the flow passage between stages of the multistage middleopen centrifugal pump is simulated, the characteristics of the flow field and the flow law are analyzed, and on the basis of the original design, the scheme of changing the shape and

\footnotetext{
*Corresponding author: 339214603@qq.com
} 
position of the guide vane is put forward, so as to achieve the purpose of improving efficiency, energy saving and emission reduction.

\section{Computation model}

\subsection{Model design}

The main design parameters of the multistage middle-open centrifugal pump are $\mathrm{Q}=$ $1.6 \mathrm{~m}^{3} / \mathrm{s}, \mathrm{H}=225 \mathrm{~m}, \mathrm{P}=5000 \mathrm{~kW}, \mathrm{n}=990 \mathrm{r} / \mathrm{min}$, efficiency $\eta=85 \%$, and the working medium is clean water. The pump adopts a horizontal center-open pump shell, with suction at both sides and middle extrusion; In order to balance the axial force to ensure the smooth operation, the impellers are symmetrically distributed from left to right. From both sides to the middle, the impellers are the first stage, the second stage and the last stage, in which the first stage impeller is single suction on both sides, and the last stage is double suction impeller. The overall structure of the pump is shown in Figure 1.

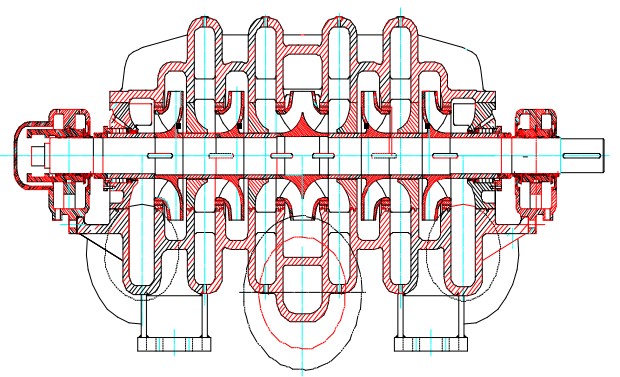

Fig.1. The structure of the Multistage pump.

The multistage pump inter-water flow channel is composed of a double volute and a bridge. The bridge refers to the part connecting the volute and the next stage impeller. The high-speed liquid produced by the impeller enters the $180^{\circ}$ symmetrical double volute ${ }^{[9]}$, and then flows through the bridge to the lower impeller. The volute of the inter-stage water flow channel is composed of two single volutes staggered by $180^{\circ}$. This structure produces the same pressure distribution on the two semicircles around the impeller. In theory, the radial forces of the impeller can offset each other. Due to the complicated structure of the inter-stage water flow channel and the difficulty in processing, a rectangular cross section can be used for the volute.

Selection of the radius of the tongue: The liquid flow at the tongue is impacted, which is prone to backflow or vortex. Reasonably selecting the radius of the tongue plays an important role in reducing the hydraulic loss. If the radius of the selected tongue is too small, it is easy to cause cavitation and accompanied by noise and vibration, which will reduce the efficiency of the pump. But if the radius of the tongue is too large, in addition to increasing the radial size of the pump casing, a certain amount of energy is also consumed, so that the efficiency of the pump decreases, so it is necessary to comprehensively consider the appropriate selection of the tongue radius. There is no special theory or empirical formula for bridge design, mainly referring to fluid flow law and empirical design.

Using Pro/E software, the three-dimensional modelling of the multistage middle-open centrifugal pump inter-stage water flow channel is modelled as shown in Figure 2. The double volutes are symmetrically distributed at $180^{\circ}$. There are two symmetrical guide 
vanes on the bridge, the main function of which is to introduce the fluid into the lower impeller.

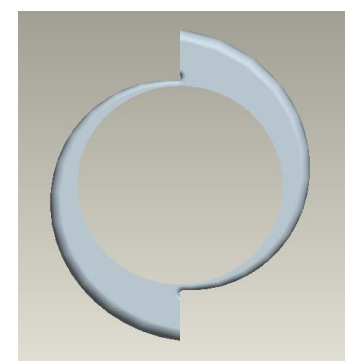

(a) double volute

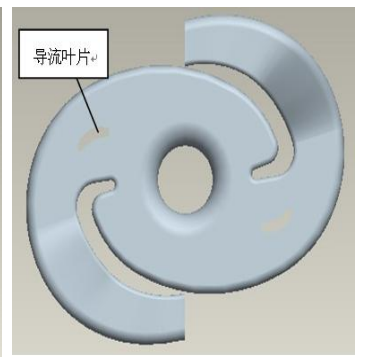

(b) the bridge

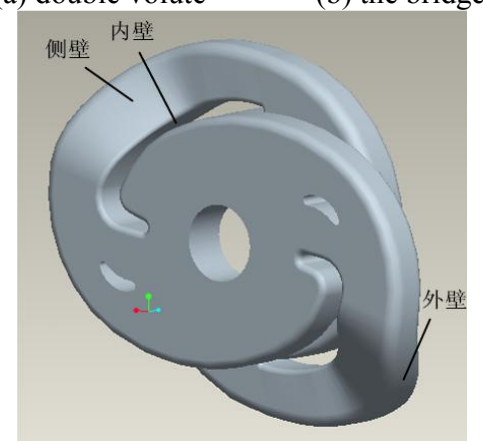

(c) Interstage water passage

Fig.2. Model graph of interstage water passage.

\subsection{Mesh generation and independence verification}

The structure of flow passage between stages of the multistage middle-open centrifugal pump is complex and twisted seriously. Therefore, unstructured grid with strong adaptability is selected for grid generation ${ }^{10-11]}$. The appropriate grid size is defined according to the degree of distortion, and the grid is encrypted in the place with a large degree of distortion and near the wall, and the grid independence is analyzed. For the convenience of calculation, the simulation uses three-stage water bodies with single suction on both sides of the first stage and double suction on the final stage.

The number of grids is approximately 2.2 million, 2.6 million, 3.2 million, 3.8 million, and 4.5 million, and the head of numerical simulation is $245.2 \mathrm{~m}, 245.9 \mathrm{~m}, 246.3 \mathrm{~m}, 246.3 \mathrm{~m}$, $246.3 \mathrm{~m}$, and the pump efficiency is $78.19 \%, 78.20 \%, 78.21 \%, 78.21 \%, 78.21 \%$. It can be seen that the calculation results of the five numbers are relatively close. Especially, the efficiency of the three grids above 3.2 million is almost consistent with the head calculation results and stable. Considering the time coordination and calculation accuracy, the number of grids can be 3.8 million, which meets the requirements, so it is selected for research.

\subsection{Governing equations}

According to the continuity hypothesis of fluid, fluid particles occupy the entire flow space continuously without gaps. The mass of the fluid is neither generated nor disappeared. That is, the increase or decrease of the mass of the fluid inside the control body is equal to 
the flow into or out of the surface the mass of the fluid, which is expressed by the differential equation as follows:

$$
\frac{\partial \rho}{\partial t}+\frac{\partial}{\partial x_{i}}\left(\rho u_{i}\right)=0
$$

Where $\rho$ is the fluid density and $u_{i}$ is the velocity component in $i$ direction.

The momentum equation is:

$$
\frac{\partial\left(\rho u_{i}\right)}{\partial t}+\frac{\partial\left(\rho u_{i} u_{j}\right)}{\partial x_{j}}=\frac{\partial p}{\partial x_{i}}+\frac{\partial}{\partial x_{j}}\left(\mu \frac{\partial u_{i}}{\partial u_{j}}\right)+\frac{\partial x_{i j}}{\partial x_{j}}
$$

Where $\tau_{\mathrm{ij}}=-\rho \mathrm{u}_{\mathrm{i}}^{\prime} \mathrm{u}_{\mathrm{j}}^{\prime}$ belongs to the Reynolds stress terms. In this paper, the standard $\mathrm{k}-\varepsilon$ ${ }^{[12]}$ model is used for numerical calculation.

\subsection{Calculation methods and boundary conditions}

The software Flunet is used for calculation and simple algorithm is used. The terms of momentum, turbulent kinetic energy and dissipation rate are in the second-order upwind format, the wall surface adopts the non-slip boundary condition the inlet section is the flow inlet while the outlet section is the static pressure one, and the dynamic-static interface model adopts Frozen rotor. The influence of roughness is considered on the solid wall and the near-wall area is processed using the standard wall surface function ${ }^{[13]}$.

\section{Calculation results and analysis}

In this paper, the multistage middle-open centrifugal pump is numerically simulated under the design conditions, and the pressure, velocity and turbulent kinetic energy distribution of the inter-stage flow passage are analyzed. The effect of the inter-stage flow passage on the pump performance is emphatically studied, and three different improvement schemes are put forward for the guide vane to optimize.

\subsection{Simulation results analysis of inter-stage flow passage}

Figure 3 shows the static pressure distribution of the impeller and the double volute section. The figure shows that the static pressure in the central section of the double volute is basically symmetrically distributed at $180^{\circ}$. The main reason for the radial force is the pressure imbalance on the coupling surface between the impeller and the volute, which due to the rotation of the impeller. The static pressure gradually increases and changes evenly from the tongue to the diffusion section. It can be seen from the static pressure distribution of the impeller in the figure that the use of symmetrical double volutes can basically offset the radial force and play a better balance. 


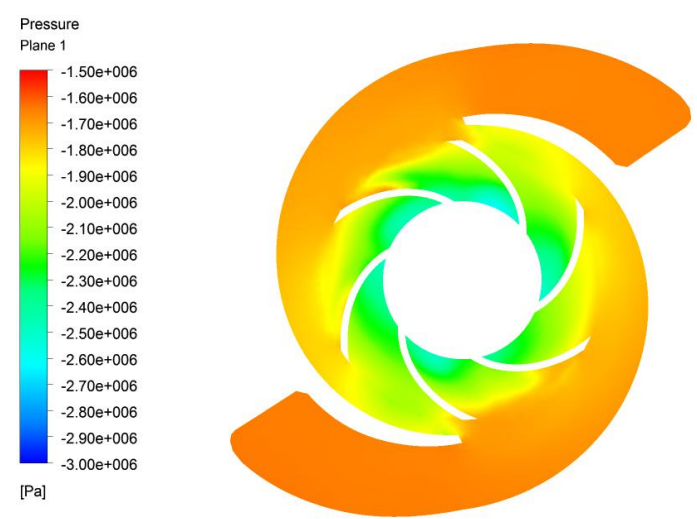

Fig.3. Static pressure profile of the impeller and the double volute.

Figure 4 shows the absolute velocity distribution near the center section and the tongue of the double volute. It can be seen in the figure that the absolute velocity of the fluid gradually decreases from the tongue to the bridge entrance, which also verifies that the volute converts the kinetic energy generated by the impeller into pressure potential energy. In addition, there are several local increase areas with $180^{\circ}$ symmetry of velocity vector near the inner ring surface of double volute, and the increase position is the same as that of static pressure reduction in Figure 3. This is because the velocity of the fluid flowing through the blade body increases when the fluid is introduced into the double volute, and a part of the potential energy becomes kinetic energy. The distribution of velocity field near the septum is reasonable and there is no obvious circumfluence and vortex, which shows that the size design of the tongue is reasonable.
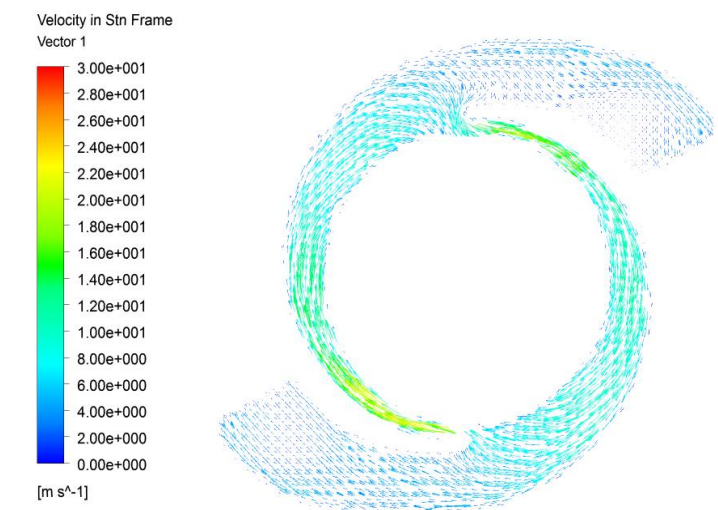

Fig.4. Absolute velocity distribution on the double volute and the volute tongue.

Figure 5 shows the static pressure distribution on the inner and outer walls of the bridge. After the water flow enters the bridge, the speed decreases, and the kinetic energy is further converted into pressure potential energy. The static pressure increases gradually along the flow direction from the bridge inlet, and the static pressure on the inner wall is smaller than that on the outer wall. The pressure fluctuates at the entrance of the bridge, which is caused by the obvious space distortion at the entrance of the bridge, resulting in the inconsistent change range of the flow direction. At the outlet of the inner and outer wall, the local pressure decreases, and the inner pressure gradient is large. This is because the inner flow channel is shorter, the distortion changes are obvious, and the sharp loss of the liquid causes the impact loss on the wall surface to increase, and the potential energy decreases; while the 
outer flow channel is longer, the transition is better, the impact loss is smaller, and the potential energy is only in a small range Inside has decreased.

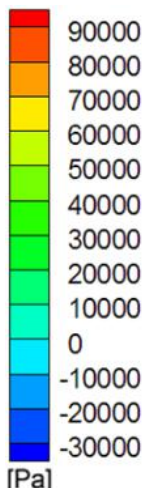

90000

80000

70000

60000

50000

40000

30000

20000

10000

$-10000$

$-20000$

$[\mathrm{Pa}]$

Fig.5. Static pressure profile on the inner and outer wall of the flow bridge.

Figure 6 and Figure 7 show the velocity distribution of the four walls of the bridge. It can be seen from the figures that the speed track at the bridge entrance is basically the same as the contour, and the speed track changes accordingly with the change of the bridge size. The space of the bridge is distorted seriously, the size changes not only in the radial direction, but also in the axial direction.
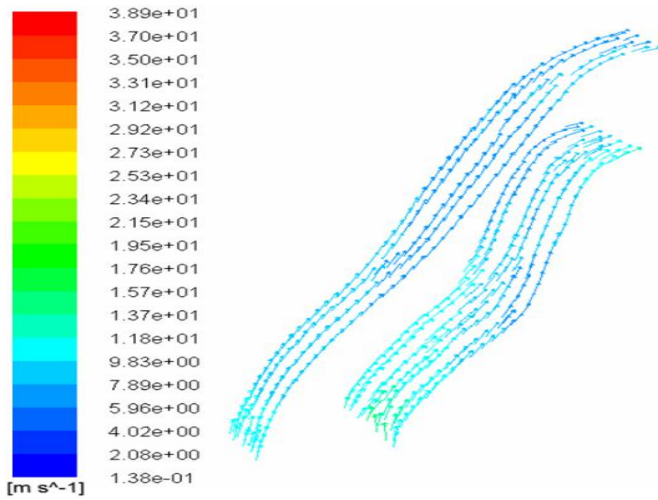

Fig.6. Velocity distribution on inner and outer wall.
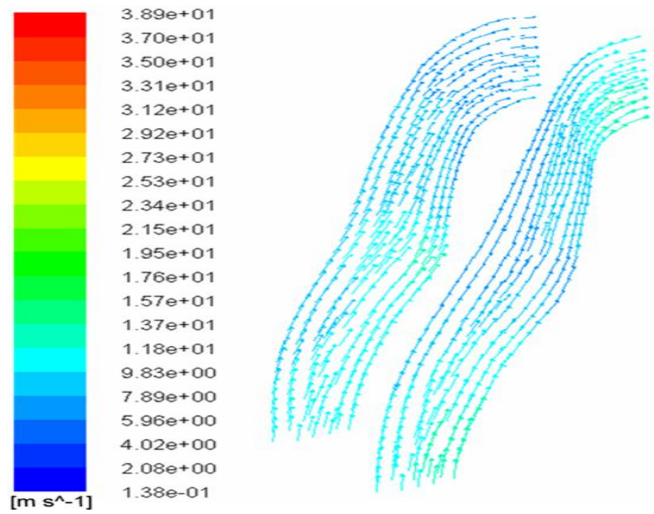

Fig.7. Velocity distribution on left and right wall. 
Figure 8 shows the turbulent kinetic energy distribution of the volute center section and the inner and outer walls of the bridge. The change of turbulent kinetic energy gradient of double volute decreases gradually along the radial direction, and the turbulent kinetic energy gradient near the septum is larger, which indicates that the energy loss is larger. The high-speed fluid in the impeller is mixed with the low-speed fluid in the volute to form a turbulent flow, which causes additional hydraulic loss, resulting in a situation where the turbulent kinetic energy gradient at the inlet of the double volute is locally large. From the whole point of view, the turbulent kinetic energy of the inner and outer wall of the bridge is relatively uniform, and only a relatively large fluctuation occurs at the inlet of the inner wall of the bridge. The main causes of the turbulent kinetic energy changes are the fluctuations of the liquid thrown out by the impeller and the impact of the liquid flow caused by the acceleration turning at the inlet of the diffusion tube.
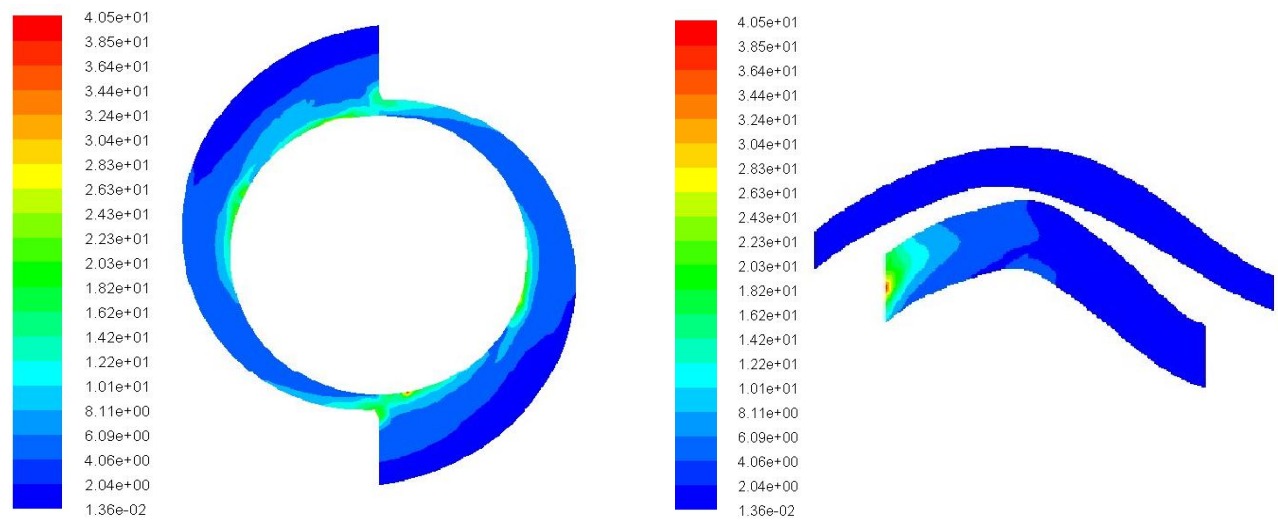

Fig.8. Turbulent kinetic energy distribution on the double volute and inner and outer wall.

Figure 9 shows the velocity vector diagram of the rear section of the bridge, where the speed trajectory is basically consistent with the contour, and the radial trajectory changes along with the speed trajectory. The outer velocity of the guide vane is larger than the inner one, that is, the kinetic energy on the outer side is larger.

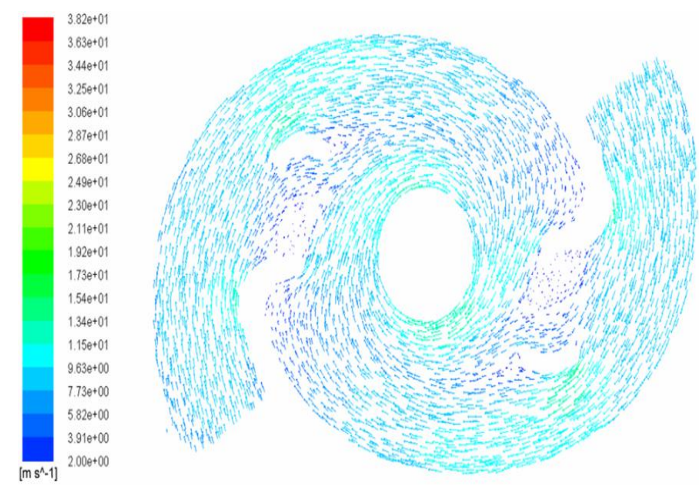

Fig.9. Velocity distribution on the trailing edge of the flow bridge.

Figure 10 shows the velocity distribution near the guide vane. As shown in the figure, the flow velocity direction of the fluid at the tip of the guide vane changes, and flow disorder occurs in a small area, besides, the flow velocity is slightly smaller than the outer side and the velocity vector line is sparse, and the phenomenon of outflow in a small area occurs. 


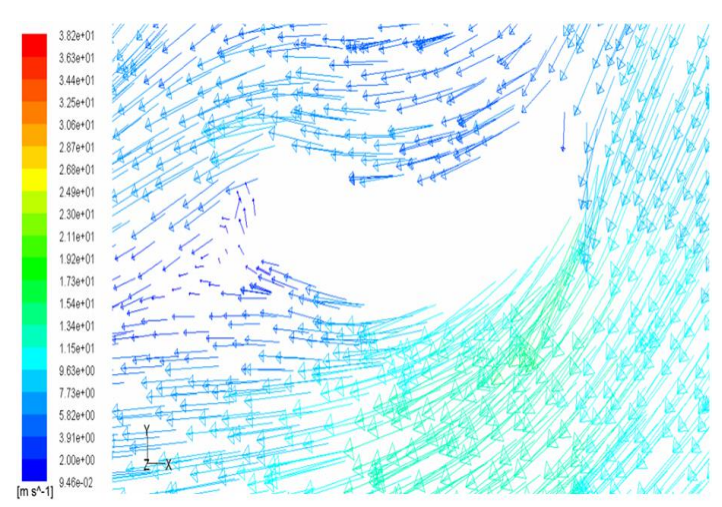

Fig.10. Velocity distribution on the guide vane.

\subsection{Structure improvement of multistage middle-open centrifugal pump inter- stage water flow channel}

Through the analysis of the inter-stage flow passage, the guide vanes on the bridge are mainly improved to improve the internal flow pattern in the flow passage, and reduce the phenomenon of backflow or vortex in it, reduce energy loss, also improve the performance of the pump. Based on the original design, by changing the angle of the front and rear ends of the guide vane in the horizontal direction ${ }^{[14-15]}$, three different improvements are proposed in table1.

Table 1. The improved schemes of guide vane.

\begin{tabular}{|c|c|c|}
\hline & $\begin{array}{c}\text { Front inlet } \\
\text { angle }\end{array}$ & $\begin{array}{c}\text { Rear exit } \\
\text { angle }\end{array}$ \\
\hline Original design & $30^{\circ}$ & $45^{\circ}$ \\
\hline Scheme 1 & $45^{\circ}$ & $45^{\circ}$ \\
\hline Scheme 2 & $60^{\circ}$ & $30^{\circ}$ \\
\hline Scheme 3 & $60^{\circ}$ & $45^{\circ}$ \\
\hline
\end{tabular}

For the above three improved schemes, the numerical simulation was carried out respectively. Through calculating the energy loss of the inter stage flow passage, the one with the least energy loss and the most reasonable design was selected.

\subsection{Simulation results analysis of the improved model}

In this paper, the multistage middle-open centrifugal pump is used. Its flow channel structure is completely symmetrical from left to right, so the structure of the flow channel between the stages is the same. Therefore, the single-stage channel on both sides is selected for numerical simulation to observe the flow condition near the guide vane, as shown in Figure 11.

Guide vanes are set in the inter-stage flow passage, which are mainly used to introduce liquid into the lower impeller. It can be seen from the flow velocity diagrams of the three improved schemes that the flow velocity in the inner side of the guide vane is less than that in the outer side, the flow velocity direction is almost the same as the shape of the vane, and only a small area of flow disorder appears at the end of the vane. Comparing the three flow velocity diagrams, it can be seen that the disorder areas of the ends of the guide vanes of the improved scheme 2 and the improved scheme 3 are smaller, which is more in line with the flow law. 


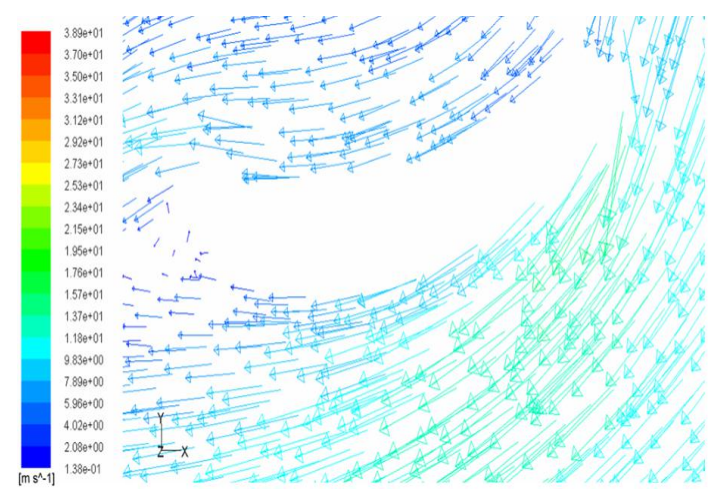

(a) Improved Scheme 1
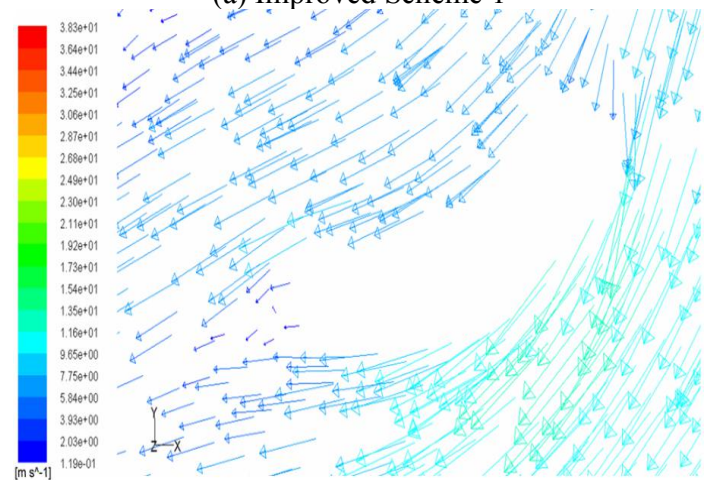

(b) Improved Scheme 2

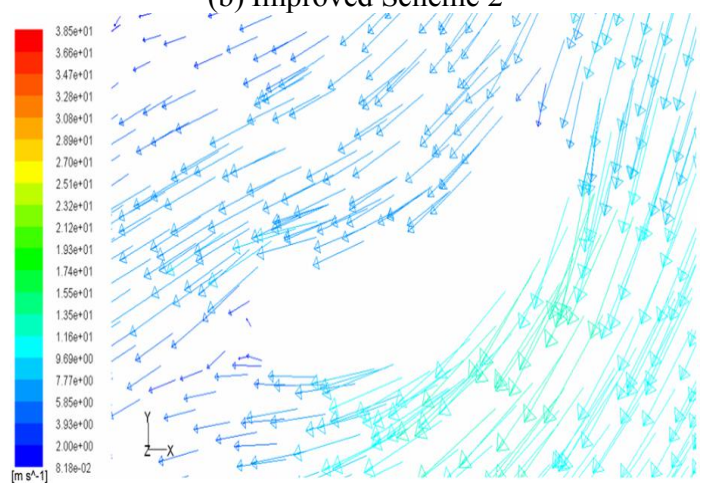

(c) Improved Scheme 3

Fig.11. Velocity distribution near the guide vane.

\subsection{Energy loss analysis of four structures}

From the perspective of energy loss, the four structures are analyzed quantitatively, and the structure with the smallest loss is finally selected. Since the fluid flows through the inter-stage flow channel inlet and outlet, following the Bernoulli equation, the energy loss at the inlet and outlet can be calculated by Fluent integral. In order to facilitate the analysis of the effect of the change of the guide vane on the performance of the multi-stage pump, the energy loss of the inter-stage water flow channel from the left is $\mathrm{h}_{\text {right, }}$ and the energy loss of the inter-stage water flow channel from the right is $h_{\text {left }}$ and the average of the two is $\mathrm{h}_{\mathrm{ave}}$, then the calculation results are shown in Table 2. 
Table 2. The energy loss comparison among different designs.

\begin{tabular}{|c|c|c|c|c|}
\hline Energy loss & Original design & Scheme 1 & Scheme 2 & Scheme 3 \\
\hline $\mathrm{h}_{\text {left }}(m)$ & 3.276 & 2.775 & 2.596 & 2.879 \\
\hline $\mathrm{h}_{\text {right }}(m)$ & 3.273 & 2.771 & 2.594 & 2.873 \\
\hline $\mathrm{H}_{\text {ave }}(m)$ & 3.2745 & 2.773 & 2.595 & 2.875 \\
\hline
\end{tabular}

According to table 2, the energy loss of the three improved schemes is less than that of the original design, and the energy loss of scheme 2 is the smallest. Therefore, the structure of scheme 2 is adopted as the final structure for further experimental and comparative research.

\subsection{Comparison of calculation and test performance curves}

The flow field of multistage pump is simulated under different conditions, and the performance parameters are predicted. The external characteristic test of water pump is carried out on the open test-bed. The main external characteristic curves of multi-stage pumps are: flow-lift characteristic curve, flow-efficiency characteristic curve and flow-axis power characteristic curve. The comparison between the test data and the calculated data is shown in Figures 12, 13, and 14.

From Figure 12, it can be seen that there is a certain error between the test value and the calculated value. The reason may be: under the design condition, there is no obvious backflow and vortex, and the flow field is relatively stable, so the experimental values and the calculated values agree well; while in the non-design conditions, the boundary layer is separated, and there are more local backflow and vortex, so the stability of the flow field is poor, resulting in large errors.

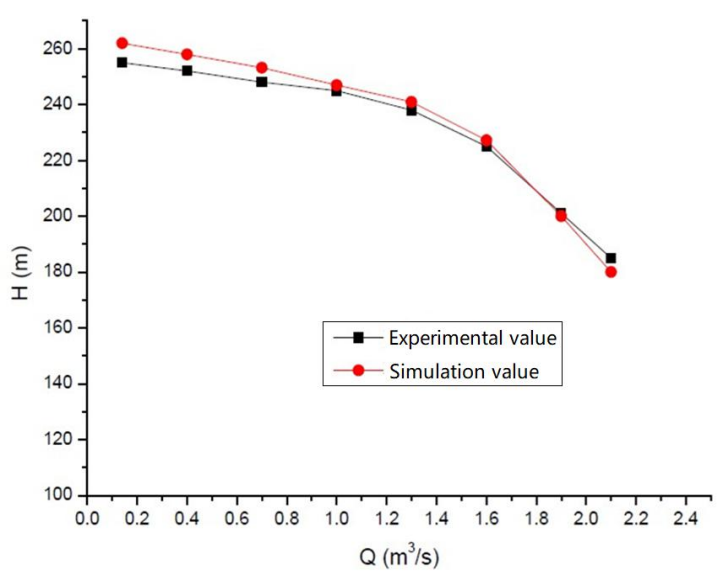

Fig. 12. The relation curve of the flow rate and head.

From Figure 13, it can be seen that the trends of the two curves are roughly the same, but the simulation calculation value is on the high side, which may be caused by the simplification of the physical model, and factors such as volume loss and clearance loss are not considered in the simulation calculation. 


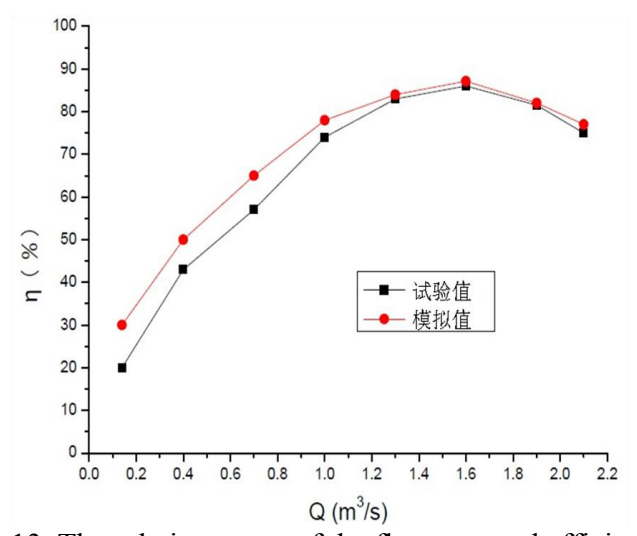

Fig.13. The relation curve of the flow rate and efficiency.

From Figure 14, it can be seen that under different flow conditions, the measured shaft power is slightly larger than the calculated one. The main reason is that the simplified physical model leads to the calculation results not including the power consumed by the loss of volume, the loss of bearing and sealing packing and the friction loss of some disks. In addition, factors such as the low roughness of the solid wall surface defined in the simulation calculation of the internal flow field of the multi-stage pump also cause the shaft power prediction curve to be lower than the test curve.

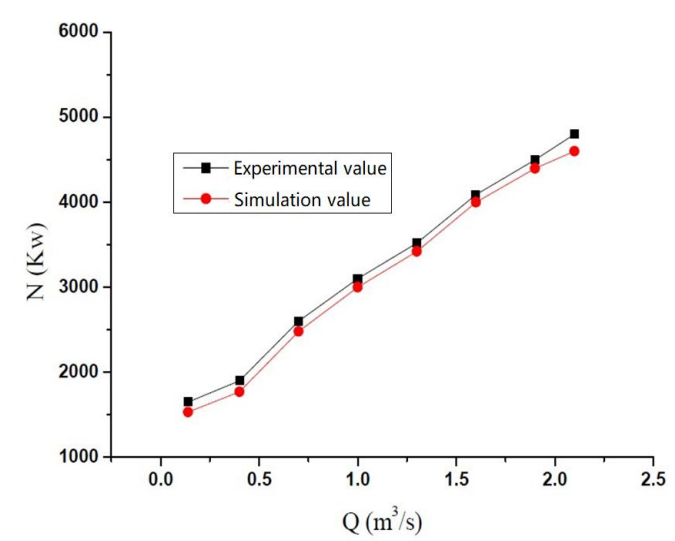

Fig.14. The relation curve of the flow rate and shaft power.

\section{3 conclusions}

(1) Based on the performance parameter requirements, this paper designs a hydraulic model of the inter-stage flow channel of the multistage middle-open centrifugal pump, reasonably matches the size of the channel between the stages, and uses Pro/E threedimensional modeling software for modeling.

(2) The numerical simulation method is used to analyze the flow velocity, pressure and turbulent kinetic energy distribution of the inter-stage flow channel. Simulation results show that the design of the double volute and the tongue is reasonable, and there are backflow and vortices at the ends of the guide vanes passing through the bridge.

(3) Aiming at the flow conditions around the guide vanes of the inter-stage flow passage, based on the original design, three different improvement schemes were proposed and 
numerical simulations were performed by changing the horizontal angle of the front and rear ends of the guide vanes, and the numerical simulation was performed to calculate the magnitude of energy loss, comprehensive analysis shows that scheme 2 has the smallest loss. Therefore, it is the optimal hydraulic model.

(4) The steady numerical simulation of the flow field of the multistage middle-open centrifugal pump is carried out under multiple working conditions, combined with experimental verification, shows that the test agrees well with the simulation and the hydraulic design meets the requirements.

\section{References}

[1] M. Liu, T. Lei, X. Yun. J. Petrol. Sci. Eng. 184 (2020)

[2] F. Lai, Y. Wang, S. A. El-Shahat, G. J. Li. J. Fluid Eng-T ASME. 141 (2019)

[3] Shah, I. R. Chuahtai. Int. J. Multiphase Flow. 37 (2011)

[4] R. Spence, J. Amaral-Teixeira. Computers \& Fluids. 37 (2008).

[5] K. Sung, K. Yi, J. H. Kim, Y. S. Choi. Adv. Mech. 11 (2019)

[6] Guo, M. Gao, J. Y. Wang, Y. T. Shi, S. Y. He. Appl. Acoust. 155 (2019)

[7] Q. R. Si, H. Y. Zhang, G. Bois, J. F. Zhang. Energies. 12 (2019)

[8] S. Huang, F. X. Yang, J. Guo. Procedia Engineering. 61 (2013)

[9] A. Panda, V. Nahornyi. Int. J. Adv. Manuf. Tech. 105 (2019)

[10] X. Q. Jia, Y. Sheng, Z. C. Zhu, B. L. Cui. Eng. Computations. (2019)

[11] T. Krzysztof, K. R. Kaczorowska-Ditrich. Flow Tureul. Combust. (2019)

[12] W. Eva, K. Mario, M. Ilona. Ann. Biomed. Eng. 48 (2019)

[13] Y. Yuan, S. Q. Yuan. Adv. Mech. 9 (2017)

[14] Y. Wang, B. F. Song, W. P. Song. J. Vibroeng. 19 (2017)

[15] J. Shendage, S. B. Kedare, S. L. Bapat. Energy. 140 (2017) 